\title{
Simultaneous structural health monitoring and vibration control of adaptive structures using smart materials
}

\author{
Myung-Hyun Kim \\ New York Power Authority, Research and Technology Development, White Plains, NY 10601, USA \\ E-mail: Myung.Kim@nypa.gov
}

\begin{abstract}
The integration of actuators and sensors using smart materials enabled various applications including health monitoring and structural vibration control. In this study, a robust control technique is designed and implemented in order to reduce vibration of an active structure. Special attention is given to eliminating the possibility of interaction between the health monitoring system and the control system. Exploiting the disturbance decoupling characteristic of the sliding mode observer, it is demonstrated that the proposed observer can eliminate the possible high frequency excitation from the health monitoring system. At the same time, a damage identification scheme, which tracks the changes of mechanical impedance due to the presence of damage, has been applied to assess the health condition of structures. The main objective of this paper is to examine the potential of combining the two emerging techniques together. Using the collocated piezoelectric sensors/actuators for vibration suppression as well as for health monitoring, this technique enabled to reduce the number of system components, while enhancing the performance of structures. As an initial study, both simulation and experimental investigations were performed for an active beam structure. The results show that this integrated technique can provide substantial vibration reductions, while detecting damage on the structure at the same time.
\end{abstract}

\section{Introduction}

Nondestructive evaluation (NDE) of structural integrity, or health monitoring, using smart materials has been of great interest to civil, ocean and space engineering community. Also, there have been a lot of research efforts incorporating smart materials as sensors and actuators for vibration suppression of flexible structures. Smart structures refer to structures with material components that can respond or adaptively change as the result of external conditions. These materials, when incorporated into structures, can provide a variety of solutions to problems associated with vibration suppression and structural diagnostics.

DeGuilio [4] proposed a technique, which allows a single piece of piezoelectric (PZT) material to concurrently control the vibration of a structure and detect structural damage. Impedance-based health monitoring techniques, which track mechanical impedance changes due to the presence of damage, have been used to assess the health condition of a structure, and multiple Positive Position Feedback (PPF) controllers have been utilized for vibration suppression. The application of this simultaneous active control and damage detection technique have certain advantages over using separate sensors and actuators for each purpose, such that the number of system components can be reduced, and that the performance of structures can be greatly enhanced. The positive position feedback (PPF) controller is a very simple and effective method for vibration suppression. However, issues related to model uncertainty and spillover were not discussed.

The impedance-based health monitoring technique is based on the change in the signature of the structural impedance. To obtain the response, a set of PZT segments are used to excite the structure and to measure the signal simultaneously. When the same PZT patches, however, are used as sensors in the control system, this signal flows into the control feedback loop. Then the 
signal may be amplified by the control gain and may cause undesirable excitations to the structure.

In this study, the feasibility of using sliding mode controller/sliding mode observer, as a part of simultaneous control and health monitoring of an adaptive structure, is presented. A sliding mode controller is designed to achieve robust vibration suppression in the presence of the modeling errors and disturbances. In particular, the use of a sliding mode observer is investigated to decouple the undesirable interaction between the health monitoring system and the control system. The key idea of this study is to use the sliding mode observer to eliminate the possibility of the undesirable excitation caused by the health monitoring system, when both health monitoring and vibration control are implemented simultaneously. This observer/control scheme is to be used in conjunction with the impedance-based structural health monitoring technique. The theory behind the techniques, both the health monitoring and the sliding mode control, is presented in the following sections.

\section{Impedance-based health monitoring technique}

The impedance-based health monitoring method utilizes the direct as well as the converse piezoelectric (PZT) effects simultaneously, hence, one PZT patch can be used for both actuation and sensing of the structural response. The basic concept of this approach is to monitor the variations in structural mechanical impedance caused by the presence of damage.

By analyzing the interaction of the PZT with the host structure, it has been derived that the electrical impedance of PZT is directly related to the mechanical point impedance of the external structure [15]. If a structure is damaged, the structural parameters, such as mass, stiffness or damping would be changed. In other words, the mechanical impedance would be modified. Since all other PZT properties remain constant, any changes in the electrical impedance signature of piezoelectric materials are attributed to damage or change in the structure. A complete description of this technique is described in the literature [12]. The variation in the electrical impedance of a PZT bonded to the structure, over a frequency range, is analogous to the frequency response but has much higher resolution and is more easily obtained. This provides a simple way of acquiring the signature pattern reflecting the current condition of the structure.

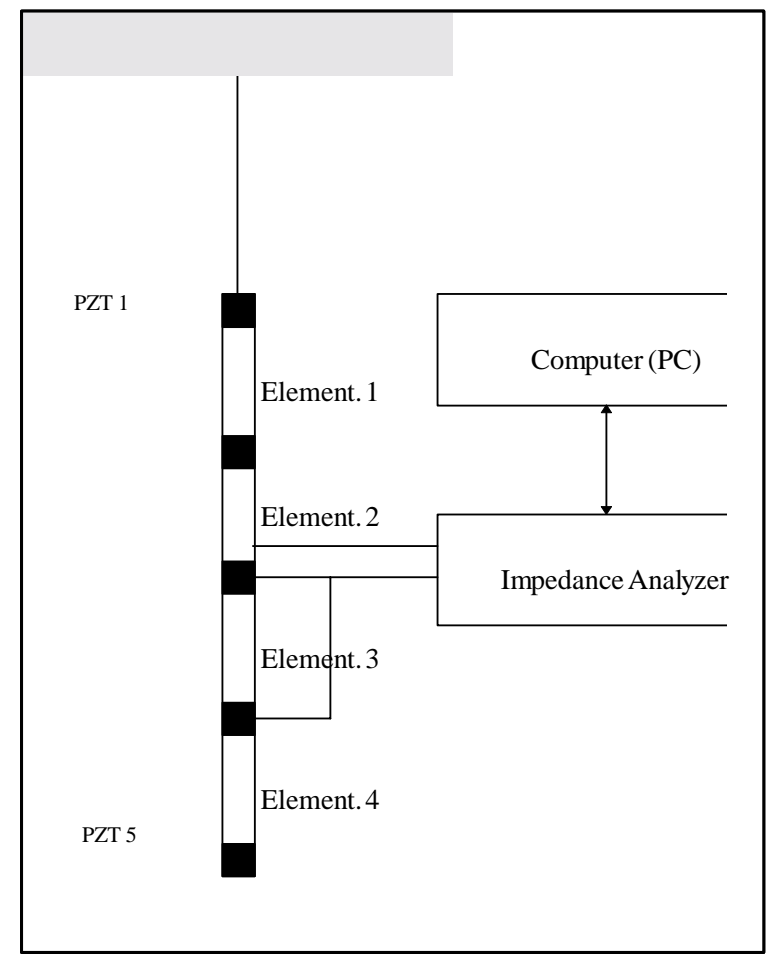

Fig. 1. Experimental setup of the aluminum bar.

Experimental implementation of the impedancebased structural health monitoring technique has been successfully conducted on several complex structures; a four bay space truss [2], an aircraft structure [3], complex precision parts [10], composite-concrete combinations [14], several built-in civil structural components [12], and a civil pipeline [13]. The method has been also tested under significant varying temperature conditions [11].

\subsection{Proof-of-concept experiment}

As a proof-of-concept application, an experiment was performed to detect and locate damage in a freefree aluminum bar, as shown in Fig. 1. The aluminum bar of dimension $630 \times 40 \times 2 \mathrm{~mm}$ was suspended by a thin wire to simulate a free-free boundary condition. Five pairs of PZT patches (Piezo Systems Inc. PSI-5H, $40 \times 15 \times 0.2 \mathrm{~mm}$ ) were bonded to the bar, for the acquisition of electrical impedance. Four elements were defined between PZT patches.

HP4194A electrical impedance analyzer was used for the measurement of the PZT's electrical impedance in the frequency range of $70-90 \mathrm{kHz}$. The range for a given structure is determined by a trial and error 


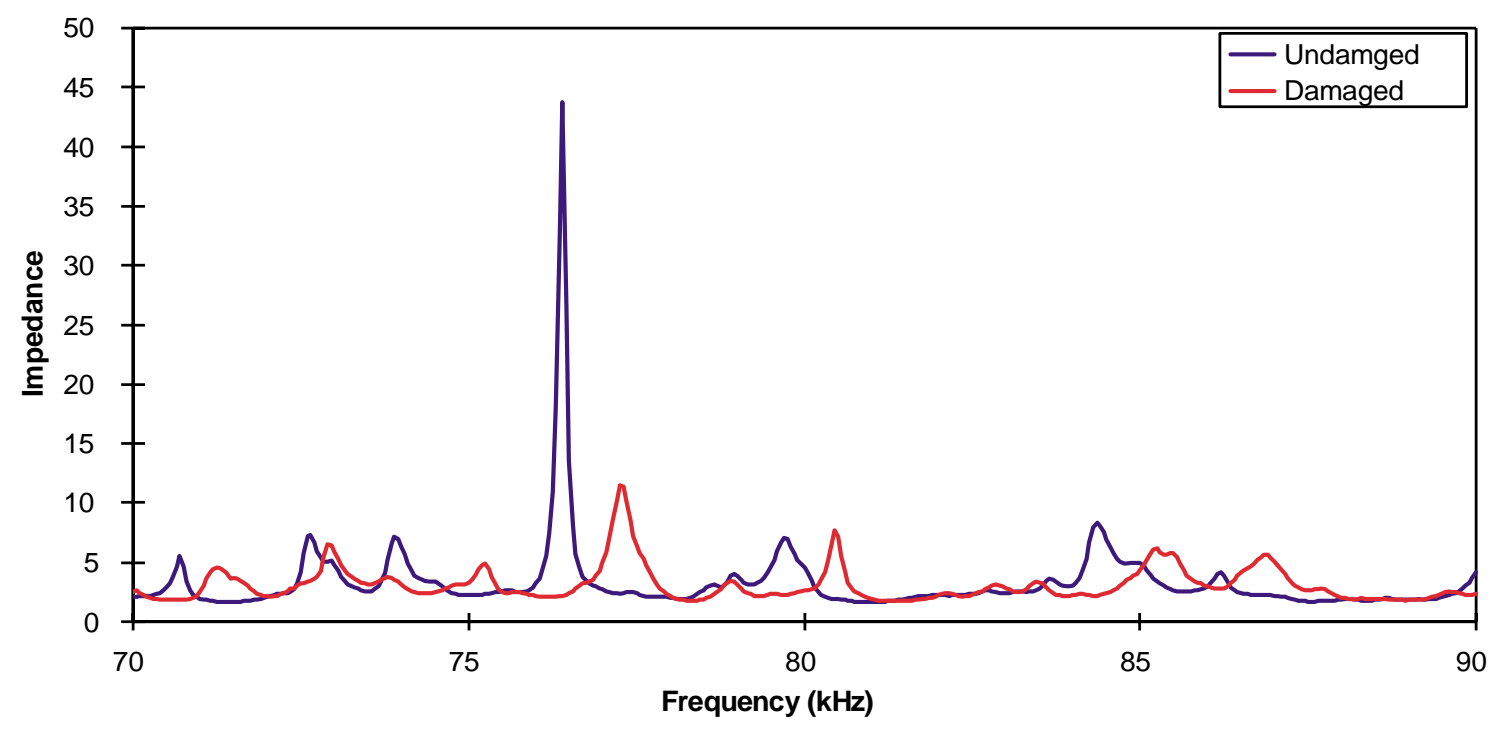

Fig. 2. Impedance measurement (real part) of PZT 3 for both undamaged and damaged cases.

method. It has been found that a frequency range with a high mode density exhibits a higher sensitivity, since it generally covers more structural dynamic information. A frequency range, $70-90 \mathrm{kHz}$, is found to be favorable in monitoring this specific structure.

Damage was induced by attaching two bolts, with diameter of $10 \mathrm{~mm}$, on the middle of the third element. This damage can be considered as an addition of mass, and also as having stiffening effects on the element by tightening bolts on the side of the cross section of the bar. The impedance measurement (real part) of the PZT 3 over the determined frequency range is shown in Fig. 2. Only the real portion of the electrical impedance is analyzed to predict damage. The real part is more sensitive to changes in structural condition than the imaginary part or magnitude, since they are dominated by the capacitive response of the sensor. As shown in Fig. 2, a complete change occurs in the impedance signature pattern over the entire frequency range and is clearly indicative of damage.

A damage metric chart is also shown in Fig. 3. The damage metric, defined as the sum of the squared differences of the real impedance changes at each frequency step, is used to simplify the interpretation of the impedance variations and provides a summary of the information obtained from each impedance response curve. As shown, there is a relatively large increase in the damage metric value for PZT's 3 and 4, indicating that the damage is located between these two sensors. This demonstrates that the damage metric chart can provide the location of damage, as well as a summary of the impedance variation.

\section{Sliding mode observer based control}

This section describes control issues related to the vibration control of flexible structures. Special attention is given to a sliding mode observer with application to vibration control problem. A sliding mode observer is initially applied to reduce observation spillover that may cause instability [9]. Another usefulness of the proposed sliding mode observer is in its robustness and disturbance decoupling property.

In theory, the dynamics of flexible structures include an infinite number of degrees of freedom [6]. For vibration controller design, however, it is common to design a control system based on only a few lower modes [1]. This makes the control bandwidth remain below a certain frequency. On the other hand, the impedance-based health monitoring method uses the signal above that frequency to ensure the sensitivity to minor changes in the near field of piezoelectric sensors. However, an interaction between the control system and the health monitoring system might occur at certain ranges of frequencies, when a relatively low frequency range is selected for health monitoring. This interaction may cause an unwanted excitation to the structure by feeding back the signal into the control loop.

Sliding mode controllers require the full state vector $[7,16]$. Usually a state estimator is designed to provide states that cannot be measured directly. Typically a Kalman filter [8] is used to decrease the elements of the observer gain matrix and consequently reduce the observation spillover due to the residual modes. How- 


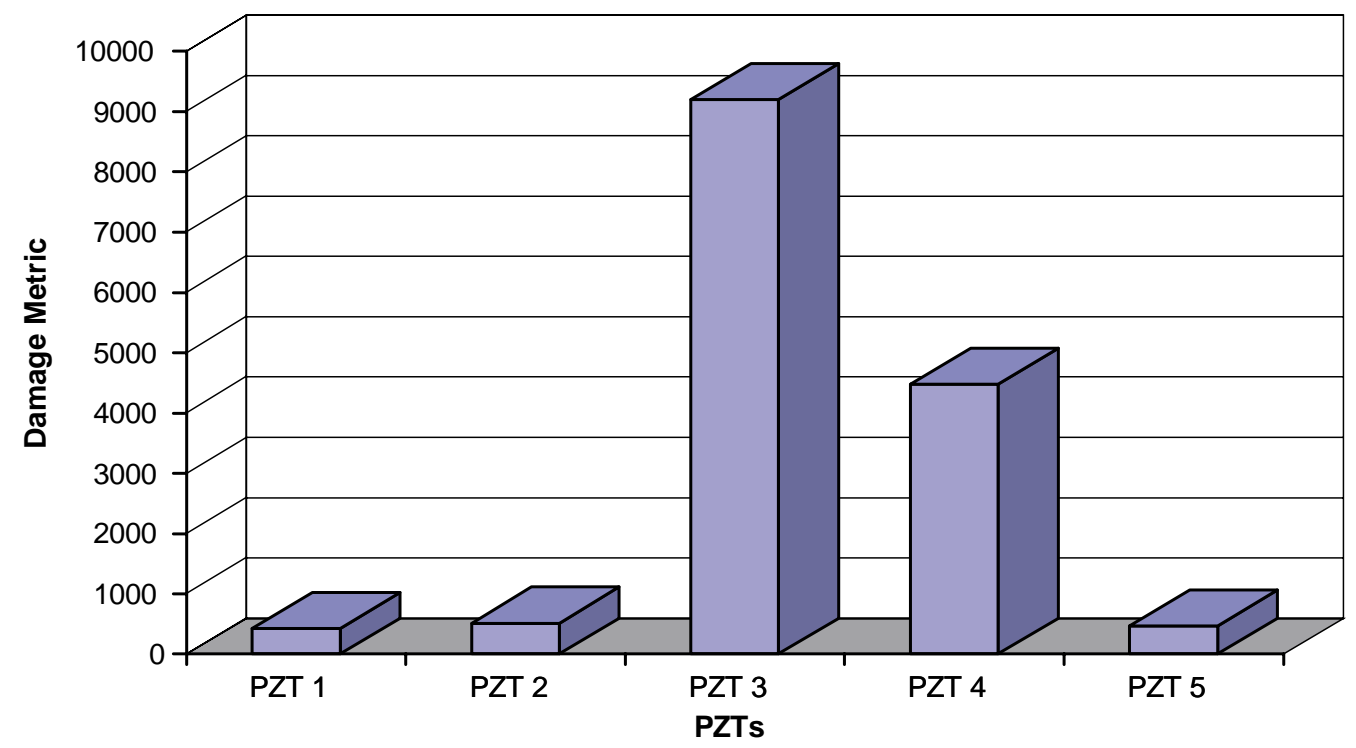

Fig. 3. Damage metric chart. The significant change in PZT 3 and 4 indicates the damage between the two sensors.

ever, these previously published results did not address the robustness issues of the controller to observation spillover. In this effort, we introduce a sliding mode controller using state estimates from a sliding mode observer that reduces the effects of observation spillover from the residual modes as well as the undesirable excitation from the health monitoring system.

\subsection{The sliding mode observer design}

The observer utilizes the same sliding mode concept as in the sliding mode control theory and is known to have the robustness property and the disturbance decoupling property. Using the properties, robust state estimation and observation spillover reduction in the presence of excitation from health monitoring system are expected. The equation of motion for flexible structures in state space form can be written as [9]

$$
\begin{aligned}
& \dot{v}_{N}=A_{N} v_{N}+B_{N} u \\
& \dot{v}_{R}=A_{R} v_{R}+B_{R} u \\
& y=C_{N} v_{N}+C_{R} v_{R}
\end{aligned}
$$

where $v_{N} \in R^{n}$ is the controlled modes, $v_{R}$ is the residual modes and $y \in R^{p}$ is the measured output. The matrices $A_{N}, B_{N}, A_{R}, B_{R}, C_{N}$, and $C_{R}$ can be defined with appropriate dimensions. Considering that the signal from the health monitoring system would flow through the control input channel, Eq. (1) can be rewritten as

$$
\dot{v}_{N}=A_{N} v_{N}+B_{N}(u+\xi)
$$

where $\xi$ is the matched disturbance from the health monitoring system and assumed to be bounded $\|\xi\| \leqslant$ $\alpha$.

The sliding mode observer is designed based on the controlled modes, Eq. (4). Introducing a nonsingular transformation $T c=\left[N_{c} C^{T}\right]^{T}$ with the columns of $N_{c} \in R^{n \times(n-p)}$ spanning the null space of $C$, the new output distribution matrix is $C T_{c}^{-1}=\left[0 I_{p}\right]$. If the other system matrices are written as

$$
T_{c} A_{N} T_{c}^{-1}=\left[\begin{array}{ll}
A_{11} & A_{12} \\
A_{21} & A_{22}
\end{array}\right] \text { and } T_{c} B_{N}=\left[\begin{array}{l}
B_{1} \\
B_{2}
\end{array}\right](5)
$$

the nominal system can be written as

$$
\begin{aligned}
& \dot{v}_{1}=A_{11} v_{1}+A_{12} y+B_{1}(u+\xi) \\
& \dot{y}=A_{21} v_{1}+A_{22} y+B_{2}(u+\xi)
\end{aligned}
$$

where $T_{c} v_{N}=\left[v_{1}^{T}, y^{T}\right]^{T}$.

The sliding mode observer proposed by Utkin [5] has the form

$$
\begin{aligned}
& \dot{\hat{v}}_{1}=A_{11} \hat{v}_{1}+A_{12} \hat{y}+B_{1} u+L \nu \\
& \dot{\hat{y}}=A_{21} \hat{v}_{1}+A_{22} \hat{y}+B_{2} u-\nu
\end{aligned}
$$

where $\left(\hat{v}_{1}, \hat{y}\right)$ represent the state estimates for $\left(v_{1}, y\right)$, $L$ is a constant observer gain matrix and $\nu$ is the discontinuous vector, which is defined later. Defining errors between the estimates and the true states, $e_{1}=\hat{v}_{1}-v_{1}$, $e_{y}=\hat{y}-y$, the error dynamics is obtained 


$$
\begin{aligned}
& \dot{e}_{1}=A_{11} e_{1}+A_{12} e_{y}-B_{1} \xi+L \nu \\
& \dot{e_{y}}=A_{21} e_{1}+A_{22} e_{y}-B_{2} \xi-\nu
\end{aligned}
$$

Define a further change in coordinates, dependent on $L$, by

$$
\tilde{T}=\left[\begin{array}{cc}
I_{n-p} & L \\
0 & I_{p}
\end{array}\right]
$$

and let $\tilde{e}_{1}=e_{1}+L e_{y}$. Then the error system with respect to the new coordinates can be written as

$$
\begin{aligned}
& \dot{\tilde{e}}_{1}=\tilde{A}_{11} \tilde{e}_{1}+\tilde{A}_{12} e_{y}-\tilde{B}_{1} \xi \\
& \dot{e_{y}}=A_{21} \tilde{e}_{1}+\tilde{A}_{22} e_{y}-B_{2} \xi-\nu
\end{aligned}
$$

where $\tilde{A}_{11}=A_{11}+L A_{21}, \tilde{A}_{12}=A_{12}+L A_{22}-\tilde{A}_{11} L$, $\tilde{A}_{22}=A_{22}-A_{21} L$ and $\tilde{B}_{1}=B_{1}+L B_{2}$.

To show the stability of the error system, choose a Lyapunov function as

$$
V=\tilde{e}_{1}^{T} P_{1} \tilde{e}_{1}+e_{y}^{T} P_{2} e_{y}
$$

with the symmetric positive definite matrices $P_{1}$ and $P_{2}$, satisfying the following relationship

$$
\begin{aligned}
& P_{1} \tilde{A}_{11}+\tilde{A}_{11}^{T} P_{1}=-Q_{1} \\
& P_{2} \tilde{A}_{22}+\tilde{A}_{22}^{T} P_{2}=-Q_{2}
\end{aligned}
$$

The time derivative of the Lyapunov function becomes

$$
\begin{aligned}
& \dot{V}=2 \tilde{e}_{1}^{T} P_{1} \dot{\tilde{e}}_{1}+2 e_{y}^{T} P_{2} \dot{e}_{y} \\
& =-\tilde{e}_{1}^{T} Q_{1} \tilde{e}_{1}-e_{y}^{T} Q_{2} e_{y}+2 \tilde{e}_{1}^{T} P_{1} \tilde{A_{12}} e_{y} \\
& -2 \tilde{e}_{1}^{T} P_{1} \tilde{B}_{1} \xi+2 e_{y}^{T} P_{2} A_{21} \tilde{e}_{1}-2 e_{y}^{T} P_{2} B_{2} \xi \\
& -2 e_{y}^{T} P_{2} \nu
\end{aligned}
$$

Now choosing,

$$
\nu= \begin{cases}\rho \frac{P_{2} e_{y}}{\left\|P_{2} e_{y}\right\|} & \text { if }\left\|P_{2} e_{y}\right\| \neq 0 \\ 0 & \text { otherwise }\end{cases}
$$

Eq. (18) can be shown

$$
\begin{aligned}
& \dot{V} \leqslant-2 \tilde{e}_{1}^{T} P_{1} \tilde{B}_{1} \xi+2 e_{y}^{T} P_{2} A_{21} \tilde{e}_{1}-2 e_{y}^{T} P_{2} B_{2} \xi \\
& +2 \tilde{e}_{1}^{T} P_{1} \tilde{A}_{12} e_{y}-2 \rho\left\|P_{2} e_{y}\right\| \\
& \leqslant 2\left\|\tilde{e}_{1}^{T} P_{1} \tilde{B}_{1} \alpha\right\|+2\left\|e_{y}^{T} P_{2} A_{21} \tilde{e}_{1}\right\| \\
& +2\left\|e_{y}^{T} P_{2} B_{2} \alpha\right\|+2\left\|\tilde{e}_{1}^{T} P_{1} \tilde{A}_{12} e_{y}\right\|-2 \rho\left\|P_{2} e_{y}\right\| \\
& \leqslant 2\left\|P_{2} e_{y}\right\|\left\{\frac{\left\|P_{1} \tilde{e}_{1}\right\|}{\left\|P_{2} e_{y}\right\|}\left\|\tilde{B}_{1} \alpha\right\|+\right. \\
& \left.\left\|A_{21} \tilde{e}_{1}\right\|+\left\|B_{2} \alpha\right\|+\left\|\frac{\tilde{e}_{1}^{T} P_{1} \tilde{A}_{12}}{P_{2}}\right\|-\rho\right\}
\end{aligned}
$$

Using Rayleigh principle, $\lambda_{\min }(Q)\|x\|^{2} \leqslant x^{T} Q x \leqslant$ $\lambda_{\max }(Q)\|x\|^{2}$,

$$
\begin{aligned}
& \dot{V} \leqslant 2\left\|P_{2} e_{y}\right\|\left\{\sqrt{\frac{\lambda_{\max }\left(P_{1} P_{1}^{T}\right)}{\lambda_{\min }\left(P_{2} P_{2}^{T}\right)}} \frac{\left\|\tilde{e}_{1}\right\|}{\left\|e_{y}\right\|}\right. \\
& \left\|\tilde{B}_{1} \alpha\right\|+\left\|A_{21} \tilde{e}_{1}\right\|+\left\|B_{2} \alpha\right\| \\
& \left.+\sqrt{\frac{\lambda_{\max }\left(P_{1} \tilde{A}_{12} \tilde{A}_{12}^{T} P_{1}^{T}\right)}{\lambda_{\min }\left(P_{2} P_{2}^{T}\right)}}\left\|\tilde{e}_{1}\right\|-\rho\right\}
\end{aligned}
$$

and choosing,

$$
\begin{aligned}
& \rho>\sqrt{\frac{\lambda_{\max }\left(P_{1} P_{1}^{T}\right)}{\lambda_{\min }\left(P_{2} P_{2}^{T}\right)}} \frac{\left\|\tilde{e}_{1}\right\|}{\left\|e_{y}\right\|}\left\|\tilde{B}_{1} \alpha\right\|+\left\|A_{21} \tilde{e}_{1}\right\| \\
& +\left\|B_{2} \alpha\right\|+\sqrt{\frac{\lambda_{\max }\left(P_{1} \tilde{A}_{12} \tilde{A}_{12}^{T} P_{1}^{T}\right)}{\lambda_{\min }\left(P_{2} P_{2}^{T}\right)}}\left\|\tilde{e}_{1}\right\|
\end{aligned}
$$

the time derivative of the Lyapunov function $\dot{V}<0$, thus $\tilde{e}_{1}$ and $e_{y} \rightarrow 0$ in finite time.

\subsection{Sliding mode controller design}

Sliding mode controller is designed to achieve a robust vibration control performance in the presence of the uncertainties and disturbances. Considering the controlled mode equation, Eq. (1), a hyperplane is defined as

$$
S=\left\{v_{N} \in R^{n}: \sigma v_{N}=0\right\}
$$

where $n$ is the number of states in the controlled modes. It is assumed that an ideal sliding motion can be induced on the hyperplane with proper control action. In addition, it can be shown that the dynamical behavior of the system on the hyperplane is insensitive to uncertainties and disturbances. The equivalent control method [5] is used, and the control action and the switching strategy are found so that an ideal sliding motion takes place on the hyperplane. Taking the time derivative of the hyperplane

$$
\dot{S}=\sigma \dot{v}_{N}=\sigma\left[A_{N} v_{N}+B_{N} u\right]=0
$$

and choosing the equivalent control action, $u_{e q}$, to be

$$
u_{e q}=-\left(\sigma B_{N}\right)^{-1} \sigma A_{N} v_{N}
$$

the closed-loop system becomes

$$
\dot{v}_{N}=\left[I-B_{N}\left(\sigma B_{N}\right)^{-1} \sigma\right] A_{N} v_{N}
$$

Assuming $\sigma B_{N}$ is nonsingular, the close-loop system can be made stable with a proper choice of $\sigma$. Several approaches can be used for the design of the hyper- 

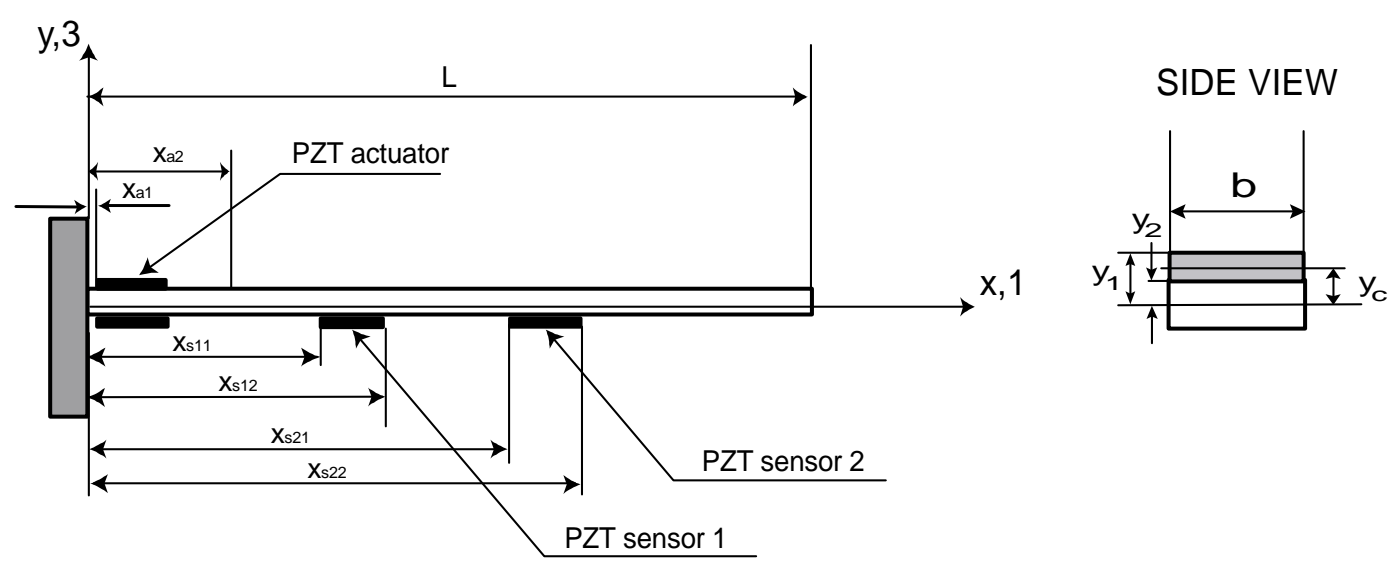

Fig. 4. Cantilever beam with PZT patches.

plane $\sigma$ such that quadratic minimization, eigenvalue placement or eigenstructure assignment methods. To achieve robustness despite uncertainties in the dynamics, a discontinuous control is added to the equivalent control. The discontinuous control has the form

$$
\bar{u}=-\rho_{c} \operatorname{sat}(S / \Phi)
$$

where $\rho_{c}$ is a positive scalar and $\operatorname{sat}(S / \Phi)$ is used to avoid the chattering phenomena due to the discontinuous control action and is defined as

$$
\operatorname{sat}(S / \Phi)= \begin{cases}\operatorname{sign}(S) & \text { if }\|S / \Phi\| \leqslant 1 \\ S / \Phi & \text { otherwise }\end{cases}
$$

Here $\Phi$ is the width of the boundary layer that is parallel to the hyperplane. The total control is composed of $u=u_{e q}+\bar{u}$.

\section{Discussion of results}

The vibration of a flexible cantilevered beam with piezoelectric (PZT) actuators/sensors, as shown in Fig. 4, is used to demonstrate the performance of sliding mode observer through the simulation as well as the experiment. A pair of PZT patches near the root of the beam is used as an actuator, and piezoelectric patches at the one-third and in the middle of the beam are used as sensors. The controller and observer are designed based on the first four lower modes of the beam. The sliding mode controller will be used in conjunction with the impedance-based health monitoring technique to provide simultaneous control and health monitoring of the structure. Robust performance of vibration suppression in the presence of uncertainties and disturbances can be achieved using the sliding mode concept.

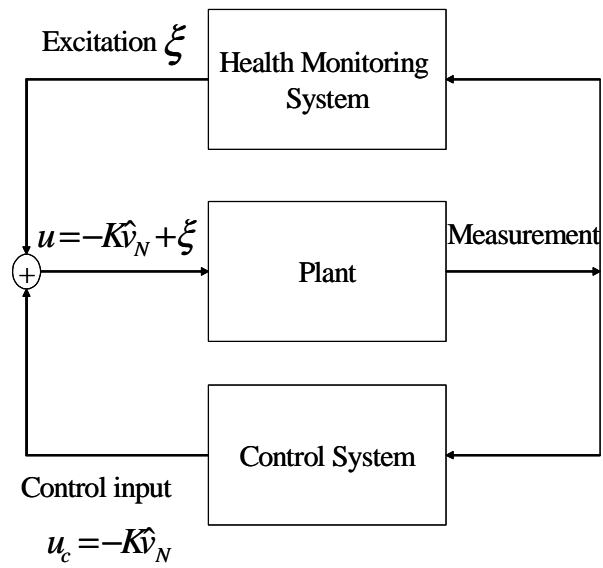

Fig. 5. The interaction of the closed-loop control system and the health monitoring system.

The closed-loop system representing the beam, the control system, and the health monitoring system is shown in Fig. 5. The details associated with the mathematical model of the beam can be found in reference [9]. The properties of the beam and PZT patches are listed in Table 1.

The state estimation performance of the sliding mode observer is compared to that of the Luenburger observer in the simulation study. A disturbance at $180 \mathrm{~Hz}$, which is close to the fifth mode of the beam, is considered to excite the beam through the control input channel. Simulation results of state estimation performances with the Luenburger observer and the sliding mode observer are shown in Figs 6 and 7, respectively. The first two states are presented in each plot in order to clearly indicate the difference in performance between two observers. As shown in Fig. 6, the Luenburger observer produces the state estimates containing the high frequency exci- 

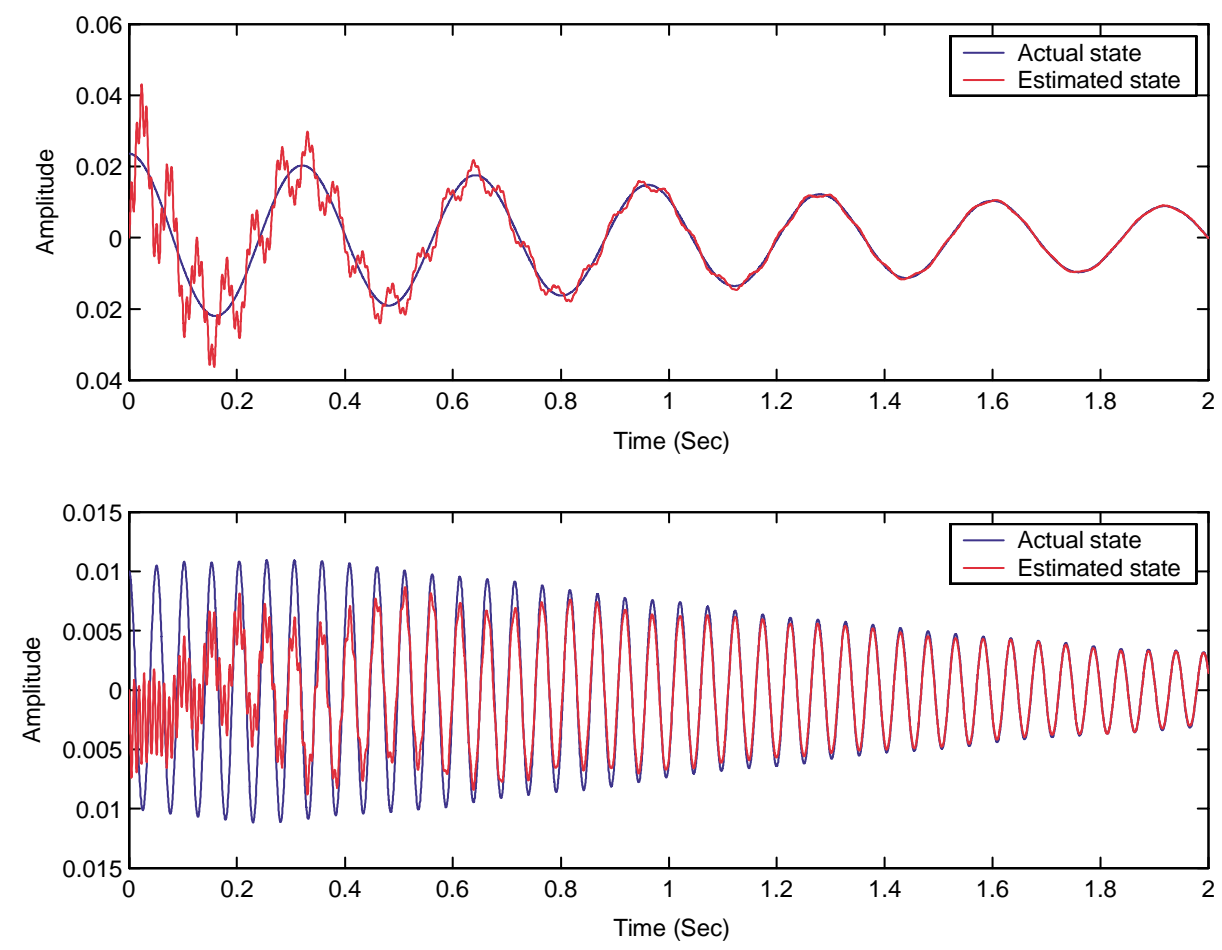

Fig. 6. State estimation performance of Luenburger observer with $180 \mathrm{~Hz}$ disturbance.
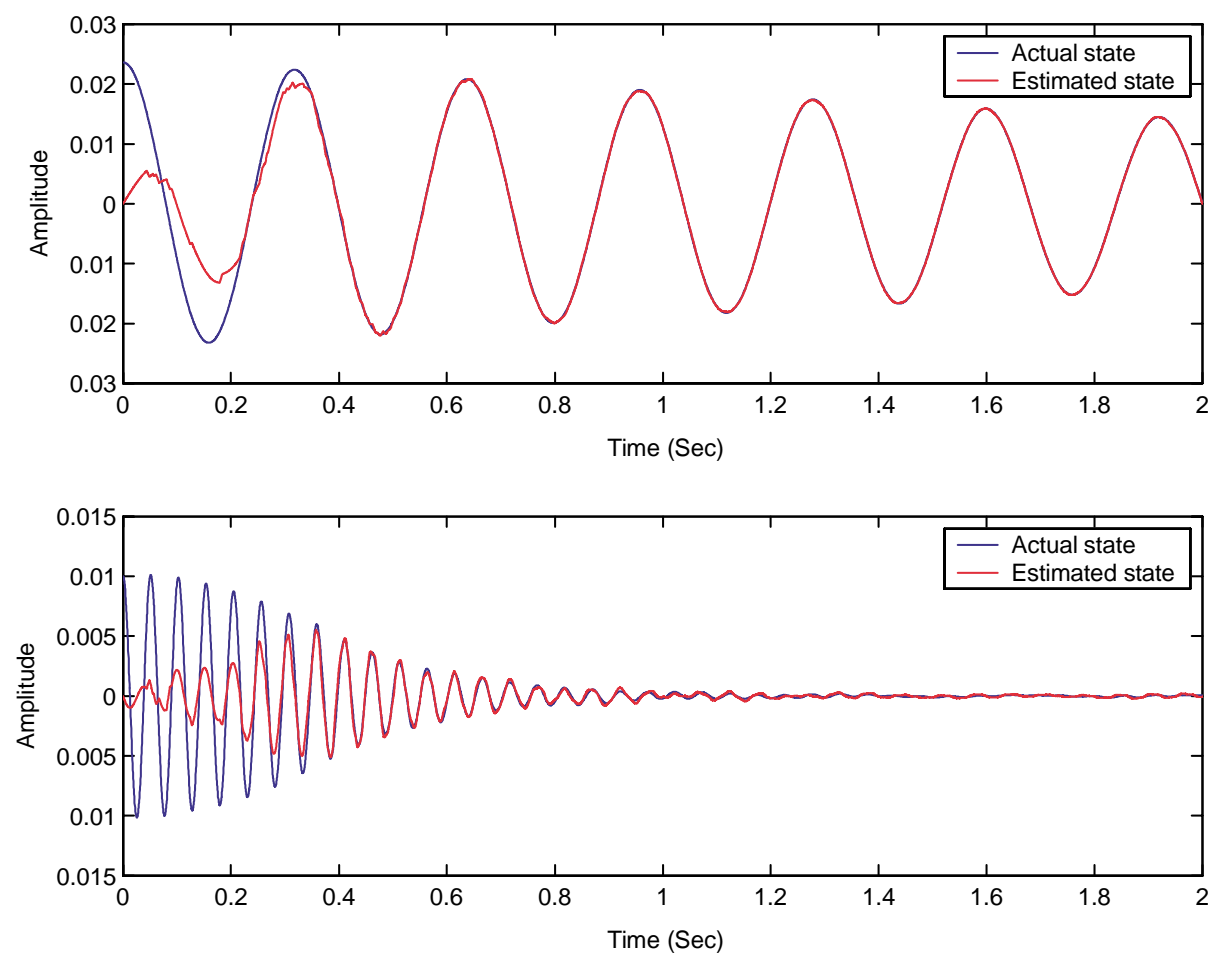

Fig. 7. State estimation performance of sliding mode observer with $180 \mathrm{~Hz}$ disturbance. 

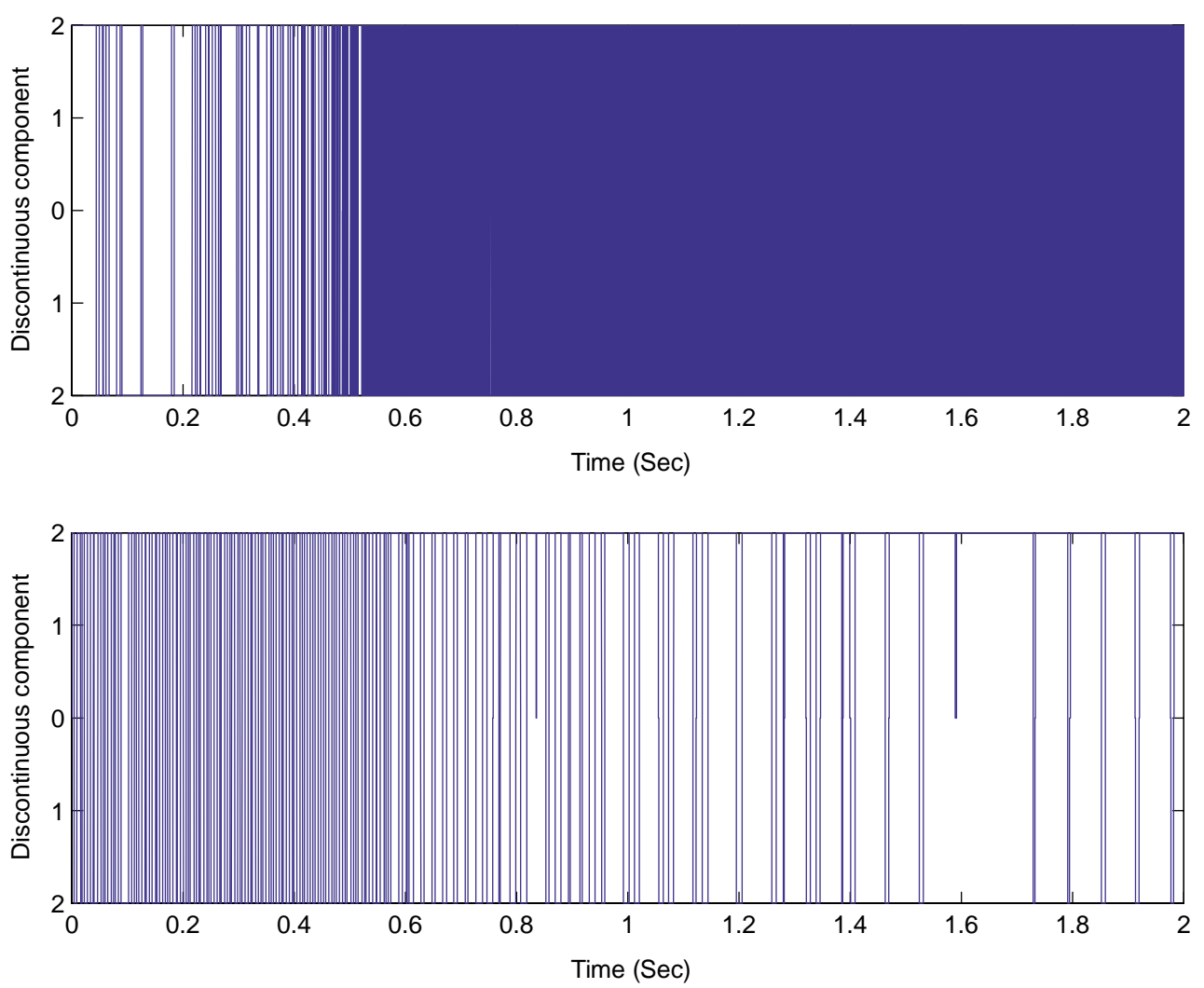

Fig. 8. Discontinuous switching components of sliding mode observer.

Table 1 Beam and PZT parameters

\begin{tabular}{lc}
\multicolumn{2}{c}{ Beam and PZT parameters } \\
\hline \multicolumn{3}{c}{ Beam parameters } \\
Length (L) & $0.63 \mathrm{~m}$ \\
Thickness (t) & $0.0012 \mathrm{~m}$ \\
Width (b) & $0.021 \mathrm{~m}$ \\
Moment of inertia (I) & $3.024 \times 10^{-2} \mathrm{~m}^{4}$ \\
Mass density $(\rho)$ & $2690 \mathrm{~kg} / \mathrm{m}^{3}$ \\
\multicolumn{2}{c}{ PZT parameters } \\
Young's modulus $\left(\mathrm{Y}_{p}\right)$ & $6.6 \times 10^{10} \mathrm{~N} / \mathrm{m}^{2}$ \\
PZT constant $\left(\mathrm{d}_{31}\right)$ & $-190 \times 10^{-12} \mathrm{~m} / \mathrm{V}$ \\
PZT capacitance $\left(\mathrm{C}_{p}\right)$ & $146 \mathrm{nF}$ \\
$\mathrm{x}_{a 1}-\mathrm{x}_{a 2}$ & $5 \mathrm{~mm}-200 \mathrm{~mm}$ \\
$\mathrm{x}_{s 11}-\mathrm{x}_{s 12}$ & $200 \mathrm{~mm}-265 \mathrm{~mm}$ \\
$\mathrm{x}_{s 21}-\mathrm{x}_{s 22}$ & $365 \mathrm{~mm}-430 \mathrm{~mm}$ \\
\hline
\end{tabular}

tation. Feeding back the state estimates with this high frequency component into the control system may excite the beam at higher modes, which is ignored in the controller design, and thus make the closed-loop system unstable. Also it can be seen that no significant vibration reduction is achieved due to the presence of the disturbance. The sliding mode observer, however, gives state estimates filtering out the high frequency excitation as shown in Fig. 7. The controller with the slid- ing mode observer achieves faster vibration suppression than with the Luneburger observer. From the simulation, we can conclude that the sliding mode observer has the desirable decoupling characteristic of filtering out the unwanted high frequency excitation from the health monitoring system. Figure 8 shows the value of the discontinuous inputs of the sliding mode observer with respect to time. The magnitudes of the discontinuous components are chosen to be 2 in this simulation study.

An experiment was performed to examine the vibration suppression performance with a sinusoidal disturbance. A dSPACE controller (dSPACE GmbH) was used in combination with Matlab real-time workshop, and the SIMULINK software was used to build the control system diagram. The sampling rate in the experiment was set at $1000 \mathrm{~Hz}$, and a signal amplifier (TREK 50/750) was used to amplify the control signal from the dSPACE controller to the PZT actuator.

Figure 9 shows an impulse response of the beam obtained from the experiment when the sliding mode controller/sliding mode observer are used for vibration control. The closed-loop response (solid) is compared 

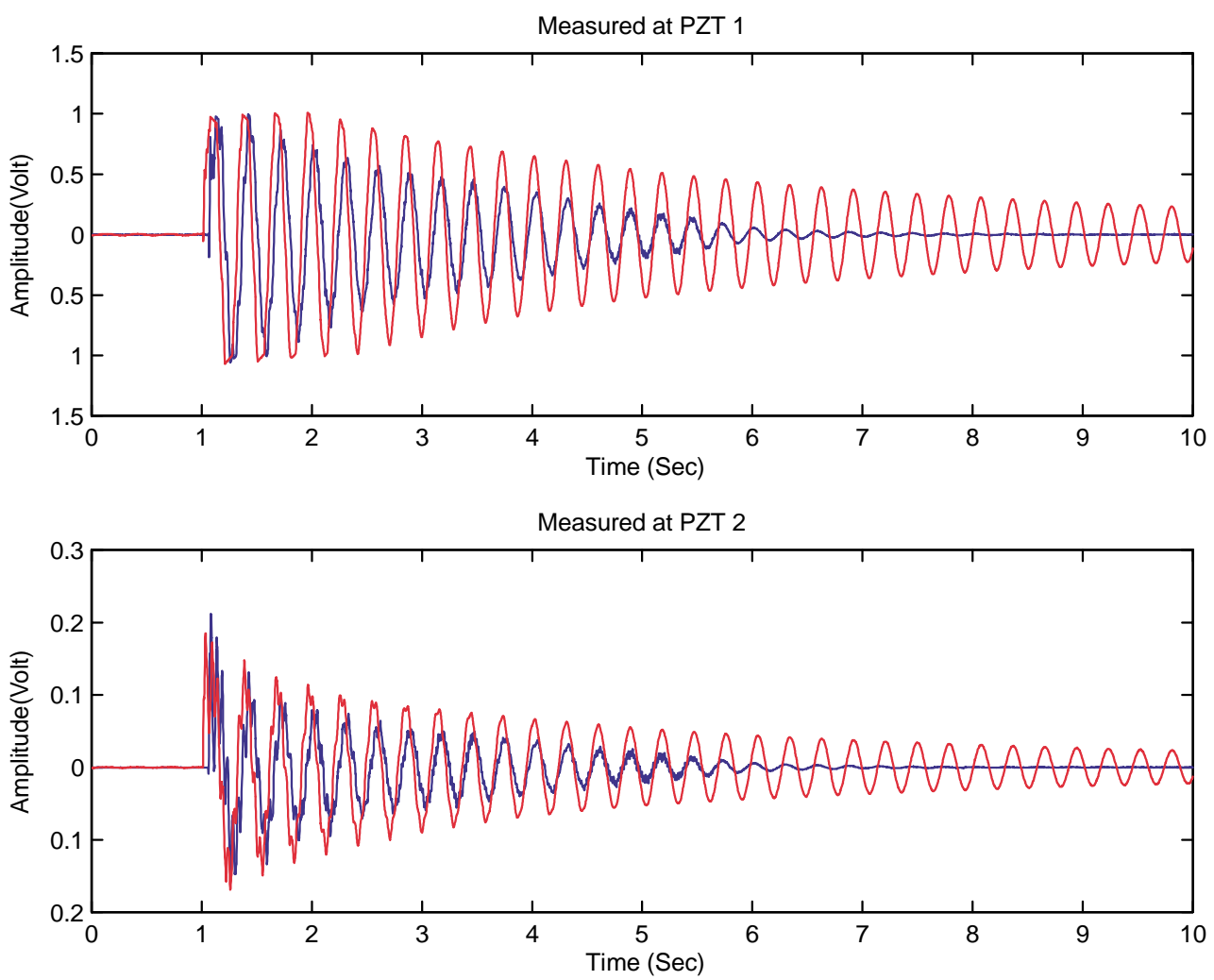

Fig. 9. The control performance of the sliding mode controller/ sliding mode observer.

to the open-loop response (dashed). An excellent vibration suppression is demonstrated, significantly reducing vibration within 5 seconds. Performances with a sinusoidal disturbance in the measurement are shown in Figs 10 and 11. Sinusoidal disturbances at $56 \mathrm{~Hz}$ with identical amplitudes were introduced through the PZT sensor 2 in two separate experiments. Figure 10 shows the control performance with $56 \mathrm{~Hz}$ (close to the second natural frequency of the beam) sinusoidal disturbance when a Luenburger observer is used for state estimation. No vibration suppression could be achieved. On the other hand, when estimates from the sliding mode observer is used for the feedback control, 78\% vibration suppression at PZT 1 and almost $90 \%$ vibration suppression at PZT 2 could be achieved, even in the presence of disturbance as shown in Fig. 11. It clearly demonstrates the effectiveness of the sliding mode observer.

\section{Conclusion}

A technique, which allows a single piece of PZT material to simultaneously control the vibration of a structure and detect structural damage, has been investigated. In particular, a sliding mode observer is proposed for state estimation to reduce the effects of unwanted high frequency excitations from the health monitoring system. A damage identification scheme, which tracks the changes of mechanical impedance due to the presence of damage, has been applied to assess the health condition of structures. As an initial study, both analytical and experimental investigations were performed to a simple beam structure. The results show that this integrated technique can provide substantial vibration reductions, while detecting damage on the structure at the same time. The proposed sliding mode observer demonstrated that it can eliminate the possible effects from high frequency excitations from the health monitoring system. In this simulation and experiment study, relatively low frequency excitations were considered. This demonstrates that the proposed technique would be useful even when different health monitoring techniques, such as the damage identification technique using modal parameters, are employed simultaneously. 

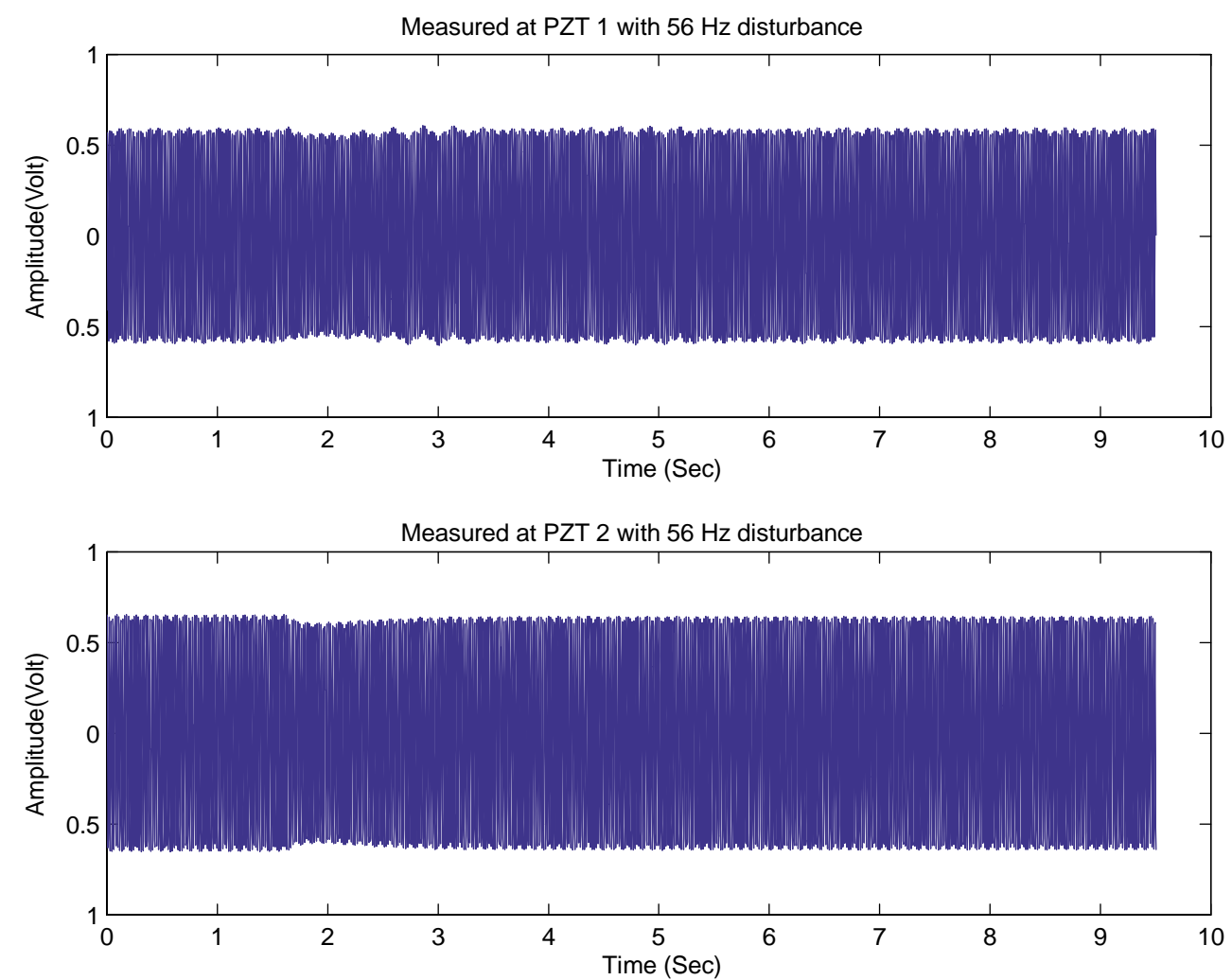

Fig. 10. Vibration suppression with $56 \mathrm{~Hz}$ sinusoidal disturbance using Luenburger observer.

\section{Acknowledgment}

This work is supported by the Office of Naval Research under grant No. N00014-96-1-1123. The author is grateful for the generous help of Professor Inman at Virginia Tech and Dr. Gyuhae Park at Los Alamos National Laboratory.

\section{References}

[1] M.J. Balas, Feedback control of flexible systems, IEEE Transactions on Automatic Control 23(4) (1978), 673-679.

[2] Z. Chaudhry, F. Sun and C.A. Rogers, Health monitoring of space structures using impedance measurements, in: Proceedings of the 5th International Conference on Adaptive Structures, Sendai, Japan, 1994.

[3] Z. Chaudhry, T. Joseph, F. Sun and C.A. Rogers, Local area health monitoring of aircraft via piezoelectric actuator/sensor patches, in: Proceedings of SPIE 1995 North American Conference on Smart Structures and Materials, San Diego, CA, 1995, vol. 2443, pp. 266-276.

[4] A. DeGuilio, Comprehensive Experimental Evaluation of Actively Controlled Piezoceramics with Positive Position Feedback for Structural Damping, Master's thesis, Virginia Polytechnic Institute and State University, Blacksburg, VA, USA, 2000 .
[5] C. Edwards and S.K. Spurgeon, Sliding Mode Control, Theory and Applications, Taylor and Francis, 1998.

[6] D.J. Inman, Active modal control for smart structures, Philosophical Transactions, Mathematical, Physical \& Engineering Sciences 359(1778) (2000), 205-219.

[7] C.K. Kao and A. Sinha, Sliding mode control of vibration in flexible structures using estimated states, in: Proceedings of the 1991 America Control Conference, 1991, vol. 3, pp. 24672474.

[8] C.K. Kao and A. Sinha, Coupled modal sliding mode control of vibration in flexible structures, Journal of Guidance, Navigation and Control 15(1) (1992), 65-71.

[9] M.H. Kim and D.J. Inman, Reduction of observation spillover in vibration suppression using a sliding mode observer, Journal of Vibration and Control 7(7) (2001), 1087-1105.

[10] F. Lalande, B. Childs, Z. Chaudhry and C.A. Rogers, High frequency impedance analysis for NDE of complex precision parts, in: Proceedings of SPIE Conference on Smart Structures and Materials, San Diego, CA, 1996, vol. 2717, pp. 237245.

[11] G. Park, K. Kabeya, H. Cudney and D.J. Inman, Impedance based structural health monitoring for temperature varying applications, JSME International Journal 42(2) (1999), 249258.

[12] G. Park, H. Cudney and D.J. Inman, Impedance-based health monitoring of civil structural components, ASCE Journal of Infrastructure Systems 6(4) (2000), 153-160.

[13] G. Park, H. Cudney and D.J. Inman, Feasibility of using impedance-based damage assessment for pipeline systems, 

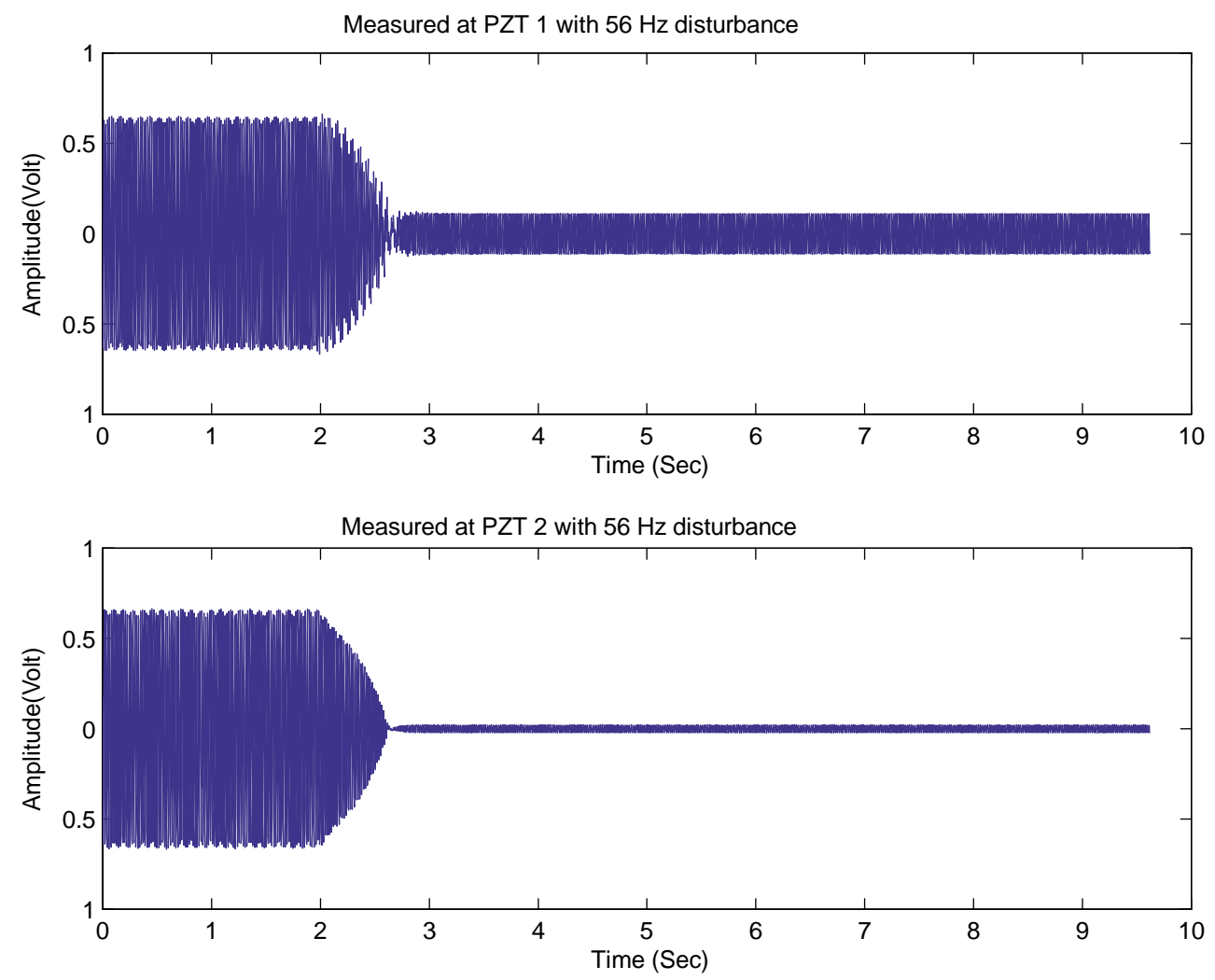

Fig. 11. Vibration suppression with $56 \mathrm{~Hz}$ sinusoidal disturbance using sliding mode observer.

Earthquake Engineering \& Structural Dynamics Journal 30 10) (2001), 1463-1474.

[14] V. Raju, G. Park and H. Cudney, Impedance based health monitoring of composite reinforced structures, in: Proceedings of 9th International Conference on Adaptive Structures and Technologies, Cambridge, MA, 1998, pp. 448-457.

[15] F. Sun, Z. Chaudhry, C. Liang and C.A. Rogers, Truss structure integrity identification using PZT sensor-actuator, Journal of Intelligent Material Systems and Smart Structures 6 (1995), 134-139.

[16] J. Tang, K.W. Wang and M. Philen, Sliding mode control of structural vibration via active-passive hybrid piezoelectric networks, in: Proceedings of SPIE The International society for Optical Engineering, vol. 3668, No. 2, 1999, pp. 543-554. 

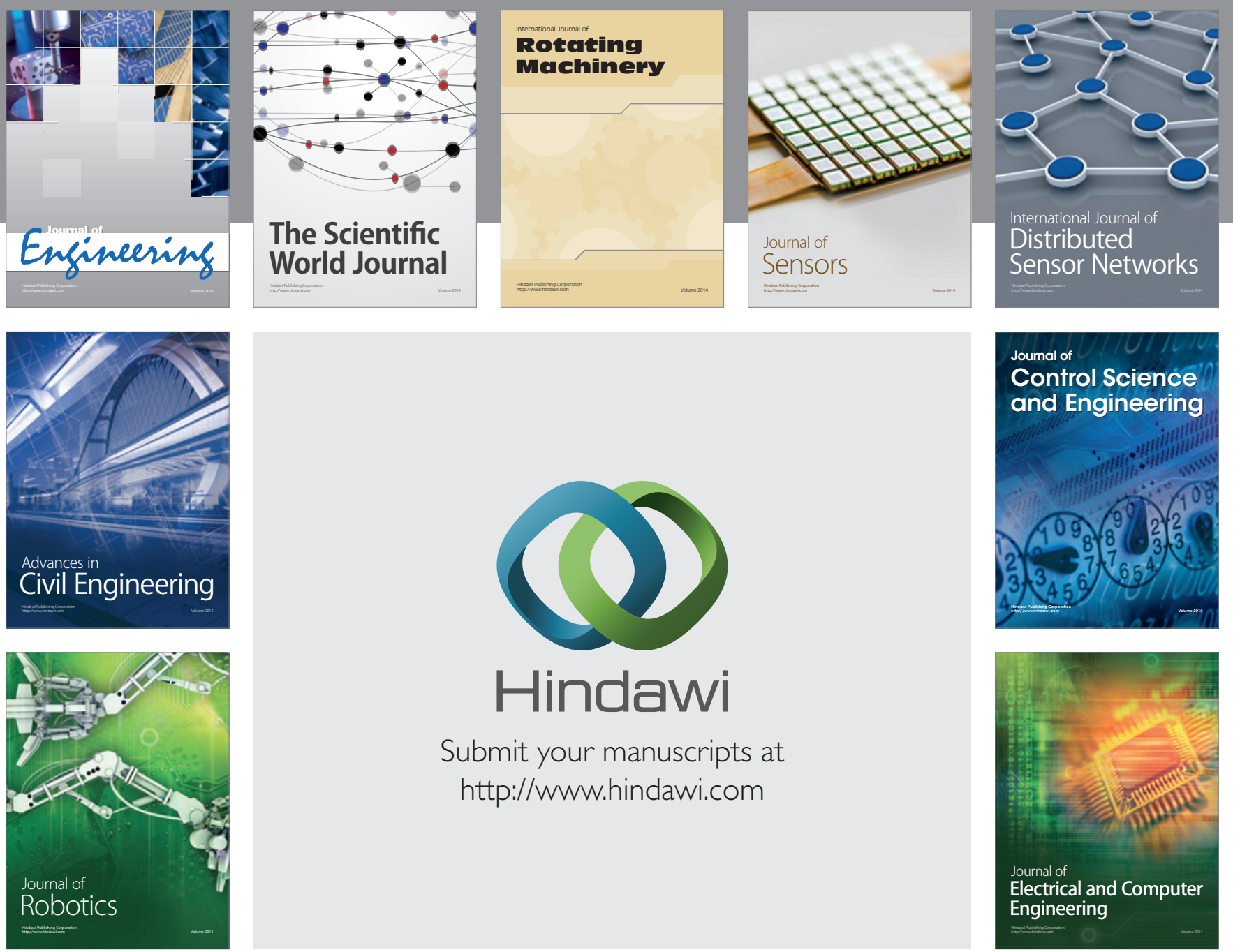

Submit your manuscripts at

http://www.hindawi.com
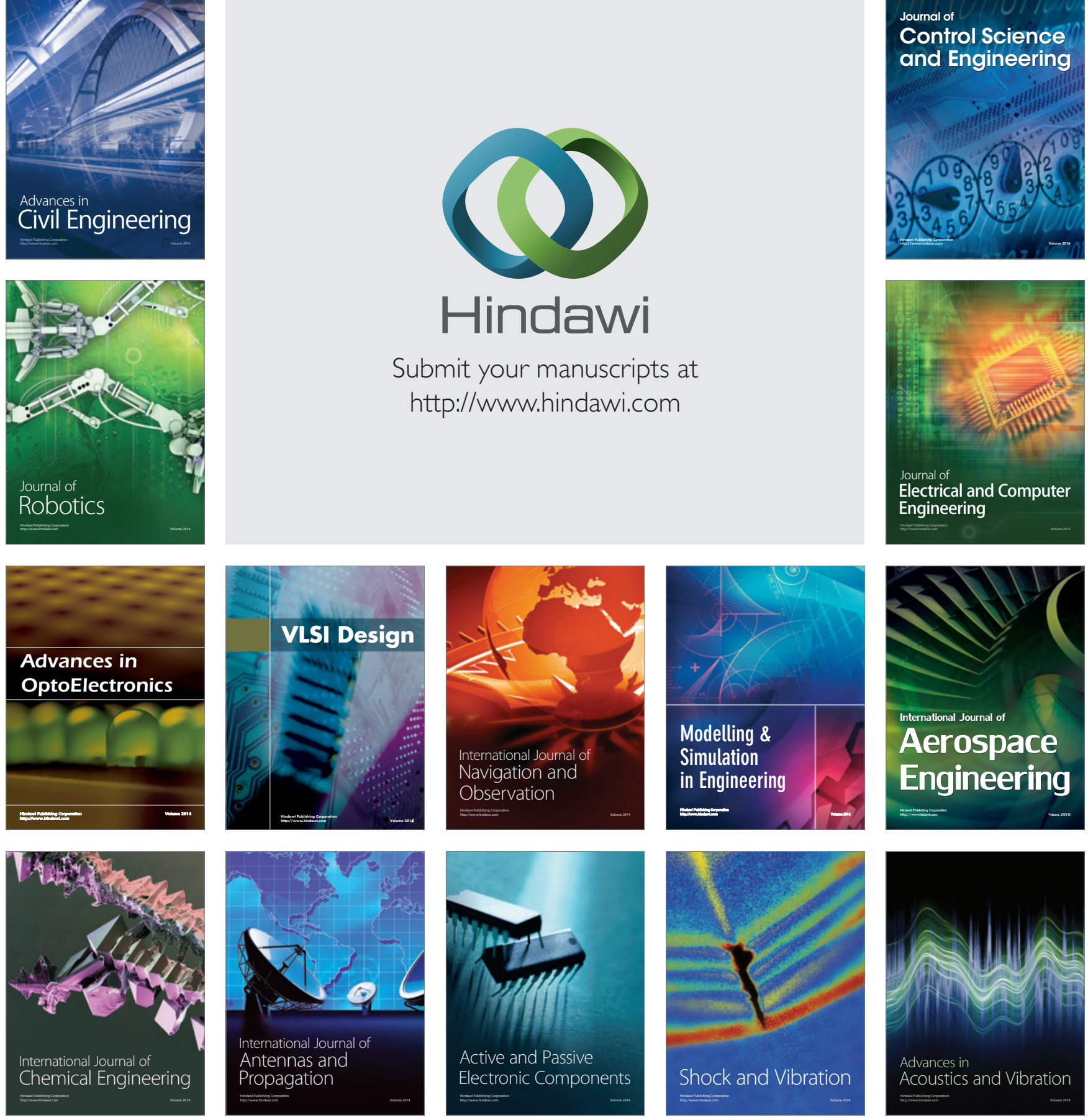